\title{
WANDERING DOMAINS FOR INFINITELY RENORMALIZABLE DIFFEOMORPHISMS OF THE DISK
}

\author{
CHRISTIAN BONATTI, JEAN MARC GAMBAUDO, JEAN MARIE LION, \\ AND CHARLES TRESSER \\ (Communicated by Charles Pugh)
}

\begin{abstract}
Denjoy's theorem and counter-example for circle maps have counterparts for infinitely renormalizable diffeomorphisms of the 2-disk.
\end{abstract}

\section{INTRODUCTION}

A series of recent results in one- and two-dimensional dynamics (cf. [S, GST]) leads us to consider here some questions inspired by Denjoy's theory [B, D], aimed at furthering our understanding of the following form of dynamics (see Figure 1 on the next page). For the orientation preserving diffeomorphism $f$ of the 2-disk:

(a) there is an invariant set $K$ which breaks into $a_{1}$ disconnected closed pieces $K\left(i_{1}\right)$ cyclically permuted by $f$,

(b) each piece $K\left(i_{1}\right)$ of $K$ breaks in $a_{2}$ disconnected closed pieces $K\left(i_{1}, i_{2}\right)$ cyclically permuted by $f_{1}=f^{a_{1}}$

(c) each piece $K\left(i_{1}, i_{2}\right)$ breaks in $a_{3}$ disconnected closed pieces $K\left(i_{1}, i_{2}, i_{3}\right)$ cyclically permuted by $f_{2}=f_{1}^{a_{2}}=f^{a_{2} \cdot a_{1}}$,

(d) continuing in this way, for all $n>0, K\left(i_{1}, \ldots, i_{n}\right)$ breaks similarly in $a_{n+1}$ disconnected closed pieces $K\left(i_{1}, \ldots, i_{n+1}\right)$ cyclically permuted by $f_{n+1}=f_{n}^{a_{n+1}}=f^{a_{n}+1} \cdot \cdots \cdot a_{1}$.

Such a map $f$ is called infinitely renormalizable. The pair $(K, f)$ is said to be of type $\left\{a_{n}\right\}_{n}$. Writing

$$
\delta_{m, \alpha}(K, f)=\sum_{0 \leq i_{j}<a_{j}}\left[\operatorname{diam}\left(K\left(i_{1}, \ldots, i_{m}\right)\right)\right]^{\alpha}
$$

for $\alpha \geq 0$, we say that $(K, f)$ has an $\alpha$-bounded geometry if

$$
\limsup _{m \rightarrow \infty} \delta_{m, \alpha}(K, f)<\infty .
$$

Received by the editors March 27, 1992 and, in revised form, December 28, 1992 and March $27,1993$.

1991 Mathematics Subject Classification. Primary 57M99, 58F99. 


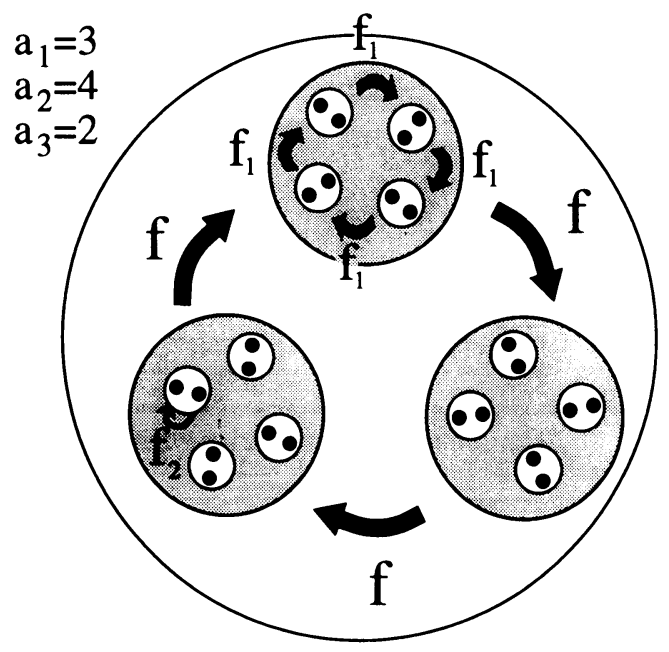

FIGURE 1

On the basis of Remark 3 below, we say that $K$ contains a wandering domain if its interior is nonvoid.

Theorem 1. Let $f$ be a $C^{1+\alpha}$ infinitely renormalizable diffeomorphism of the 2disk. If $(K, f)$ has $\alpha$-bounded geometry, with $0 \leq \alpha \leq 1$, then each connected component of $K$ has zero Lebesgue measure. In particular, $K$ does not contain any wandering domain.

Theorem 2. For any sequence $\left\{a_{n}\right\}_{n}$ with $a_{n} \geq 2$, there exists a $C^{1}$ infinitely renormalizable diffeomorphism $f$ of the 2-disk such that $(K, f)$ is of type $\left\{a_{n}\right\}_{n}$, with a 1-bounded geometry, and $K$ contains a wandering domain.

Remark 3. In the classical Denjoy theory, a wandering domain is a fibre with nonempty interior, of a semiconjugacy $h$ from a homeomorphism $f$, of the circle $\mathbb{T}$, to a translation $R_{\alpha}$ of $\mathbb{T}$, with irrational angle $\alpha$. This means that $h: \mathbb{T} \rightarrow \mathbb{T}$ is a continuous onto map such that $h \circ f=R_{\alpha} \circ h$ and, for some $x \in \mathbb{T}$, the set $h^{-1}(x)$ has nonempty interior, and we notice that the irrationality of $\alpha$ prevents the motion to be periodic. Our use of the words "wandering domain" involves two generalizations:

(1) the model map is a nonperiodic translation $T$ on a compact abelian group $G$ which is not necessarily the circle (see Remark 4), ${ }^{1}$

(2) if $f$ is a homeomorphism of $M$, the semiconjugacy is not necessarily from $f$ to $T$ but from the restricted map $f_{\mid N}$ to $T$ for some invariant subset $N \subset M$.

Remark 4. In our case, with the notation of Remark 3,

- $G$ is $\widehat{\mathbb{Z}}_{Q}=\lim _{\leftarrow q_{i}} \mathbb{Z} / q_{i} \mathbb{Z}$ where $Q$ stands for a super natural number $Q=\prod_{p} p^{k_{p}}$ where, $\forall p$ prime, $0 \leq k_{p} \leq \infty$, and the $q_{i}$ 's form a sequence of divisors of $Q$ ordered by divisibility, and

- $T$ is adding 1 on $G=\widehat{Z}_{Q}$, i.e., a generalized adding machine, where the usual adding machine corresponds to the case when $a_{n} \equiv 2$.

\footnotetext{
1 This might be further generalized to other recurrent aperiodic motions.
} 


\section{Proof of Theorem 1}

A connected component $D_{\infty}$ of $K$ is the intersection of an infinite sequence of nested $K\left(i_{1}, \cdots, i_{m}\right)$ 's. Actually, we can always arrange the labels so that

$$
D_{\infty}=\bigcap_{m \geq 0} K \underbrace{(0, \ldots, 0)}_{m \text { times }} .
$$

Assume $\int_{D_{\infty}} d x \neq 0$, and let

$$
u_{n}=\int_{D_{\infty}} \operatorname{det} D\left(f^{n}\right)(x) d x \text { and } v_{n}=\int_{D_{\infty}} \operatorname{det} D\left(f^{-n}\right)(x) d x .
$$

Since $D_{\infty}$ never intersects its images under iteration, we have $u_{n} \rightarrow 0$ and $v_{n} \rightarrow 0$ as $n \rightarrow \infty$. It follows that

$$
\int_{D_{\infty}} \log \left(\operatorname{det} D\left(f^{n}\right)(x)\right) d x \rightarrow-\infty \quad \text { when } n \rightarrow \pm \infty
$$

and thus

$W_{n}=\sum_{i=0}^{n-1} \int_{D_{\infty}}\left[\log \left(\operatorname{det} D(f)\left(f^{i}(x)\right)\right)-\log \left(\operatorname{det} D(f)\left(f^{i-n}(x)\right)\right] d x \rightarrow-\infty\right.$ as $n \rightarrow \infty$.

Assume that $n=a_{1} \cdots \cdots a_{m}$. Then for $0 \leq i \leq n-1, f^{i}\left(D_{\infty}\right)$ and $f^{i-n}\left(D_{\infty}\right)$ belong to the same $K\left(i_{1}, \ldots, i_{m}\right)$, while $f^{j}\left(D_{\infty}\right)$ belongs to a different $K\left(i_{1}, \ldots, i_{m}\right)$ for $j \notin\{i, i-n\}$ (see Figure 2).

If $f$ is $C^{1+\alpha}$, then there exists some $C>0$ such that, for all $y$ and $z$ in $\mathbb{D}^{2}$

$$
|\log (\operatorname{det} D f(y))-\log (\operatorname{det} D f(z))| \leq C \cdot|y-z|^{\alpha}
$$

so that $\left|W_{n}\right| \leq C \cdot \delta_{m, \alpha}(K, f) \cdot \int_{D_{\infty}} d x$. Thus, if $(K, f)$ has $\alpha$-bounded geometry, $\left|W_{n}\right|$ cannot go to $\infty$ as $n \rightarrow \infty$, a contradiction.

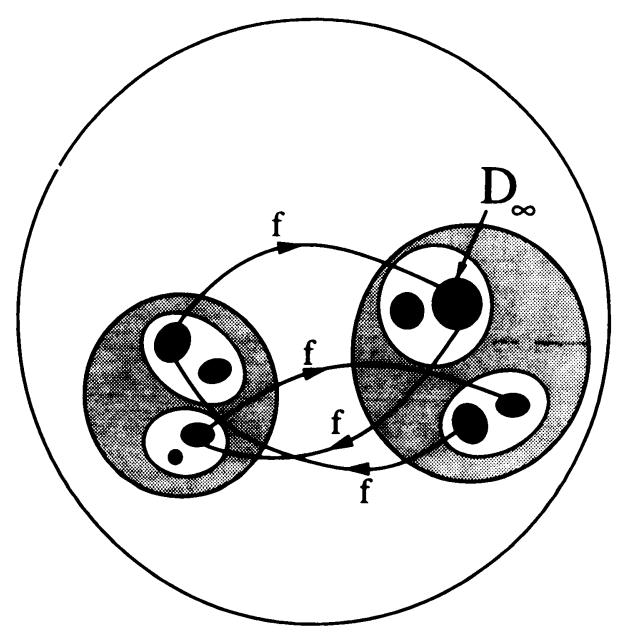

FIGURE 2 


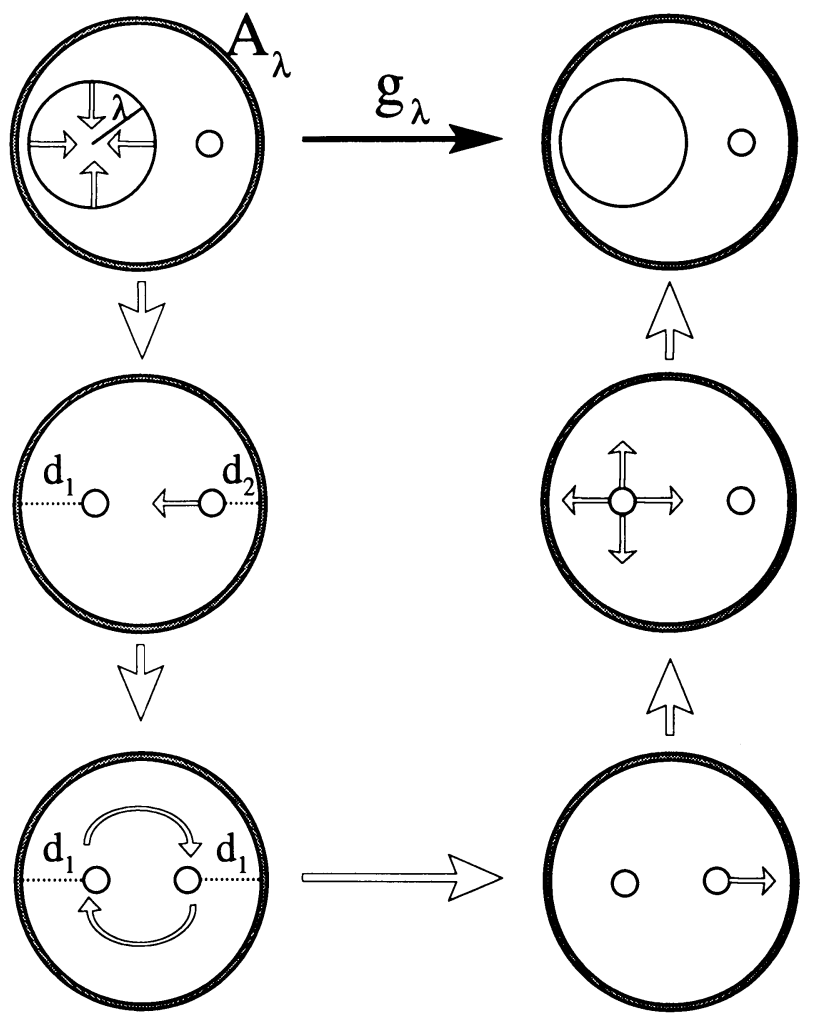

Figure 3

\section{Proof of Theorem 2}

To simplify the formulas and the picture, we shall restrict here to the case when $a_{n} \equiv 2$. We are going to construct a map $f$ as the limit in the $C^{1}$ topology (with norm $\|\cdot\|_{1}$ ) of a sequence of $C^{\infty}$ orientation-preserving diffeomorphisms $\left\{f_{n}\right\}_{n}$ of the 2-disk $\mathbb{D}^{2}$. This construction is merely a modification of the one in $[\mathrm{BF}]$.

As the main building block of the construction, let us consider a smooth orientation-preserving diffeomorphism $g_{\lambda}$ of $\mathbb{D}^{2}$, where $\frac{1}{2} \leq \lambda<1$, with the following properties (see Figure 3):

- $g_{\lambda}$ is the identity in the annulus $\mathscr{A}_{\lambda}=\left\{x \in \mathbb{D}^{2} \mid\|x\|>\frac{5+3 \lambda}{8}\right\}$,

- $\mathbb{D}^{2} \backslash \mathscr{A}_{\lambda}$ contains two disjoint disks, $D_{\lambda}$ with radius $\lambda$ and $D_{\lambda}^{\prime}$ with radius $\frac{1-\lambda}{4}$, such that $g_{\lambda}$ maps each of these inner disks to the other as a composition of homotheties, translations, and rotations.

We regroup the following statements in the form of a lemma, leaving the details of the proof to the reader (see Figure 3):

Lemma. We can choose the family of maps $\left\{g_{\lambda}\right\}_{\lambda \in[1 / 2,1)}$, and for each $\lambda$ an isotopy $g_{\lambda}^{t}$ from the identity $\mathrm{Id}_{\mathbb{D}^{2}}$ to $g_{\lambda}$ so that:

(i) $g_{\lambda}^{t}$ is the identity in $\mathscr{A}_{\lambda}$,

(ii) $g_{\lambda}^{t}$ restricted to both $D_{\lambda}$ and $D_{\lambda}^{\prime}$, is a composition of homotheties, translations, and rotations, 
(iii) there exists a constant $K>0$ such that $\left\|g_{\lambda}-\operatorname{Id}_{\mathbb{D}^{2}}\right\|_{1} \leq K /(1-\lambda)$ and $\left\|g_{\lambda}^{(i+1) / N} \circ\left(g_{\lambda}^{i / N}\right)^{-1}-\mathrm{Id}_{\mathbb{D}^{2}}\right\|_{1}$ $\leq \frac{K}{N \cdot(1-\lambda)}$ for all $\lambda$ in $\left[\frac{1}{2}, 1\right), N>0$, and $0 \leq i<N$.

Now choose a sequence $\left(\lambda_{m}\right)_{m>0}$, with $\lambda_{m} \rightarrow 1$, and a sequence of affine scalings $\left(\Lambda_{m}\right)_{m>0}$ such that $\Lambda_{m}$ maps $D_{\lambda_{m}}$ onto $\mathbb{D}^{2}$. We also define the affine scalings $A_{m}$ and the disks $D_{m}$ by

$$
A_{m}=\Lambda_{1} \circ \cdots \circ \Lambda_{m} \text { and } D_{m}=A_{m}^{-1}\left(\mathbb{D}^{2}\right) \text {. }
$$

Everything is now in place for the construction. We set $f_{1}=g_{\lambda_{1}}$ and define inductively the sequence $\left\{f_{n}\right\}_{n}$ by setting

$$
f_{m+1}= \begin{cases}f_{m}^{i+1} \circ A_{m}^{-1} \circ g_{\lambda_{m+1}}^{(i+1) / 2^{m}} \circ\left(g_{\lambda_{m+1}}^{i / 2^{m}}\right)^{-1} \circ A_{m} \circ f_{m}^{-i} & \text { for } x \in f_{m}^{i}\left(D_{m}\right), \\ f_{m} \text { elsewhere. } & i=0, \ldots, 2^{m}-1,\end{cases}
$$

The map $f_{m}$ is a $C^{\infty}$ diffeomorphism that cyclically permutes $2^{m}$ disks $f_{m}^{i}\left(D_{m}\right)$ for $i=0, \ldots, 2^{m}-1$ and the restriction of $f_{m}^{2^{m}}$ to $D_{m}$ is the identity.

The definition of $f_{m+1}$ is such that it differs from $f_{m}$ only on the disks $f_{m}^{i}\left(D_{m}\right)$, where

$$
f_{m+1}-f_{m}=f_{m}^{i+1} \circ A_{m}^{-1} \circ\left(g_{\lambda_{m+1}}^{(i+1) / 2^{m}} \circ\left(g_{\lambda_{m+1}}^{i / 2^{m}}\right)^{-1}-\operatorname{Id}_{\mid \mathbb{D}^{2}}\right) \circ A_{m} \circ f_{m}^{-i},
$$

and since the restriction of $f_{m}$ to the disks $f_{m}^{i}\left(D_{m}\right)$ is a composition of homotheties, translations, and rotations, it follows from $(*)$ and the lemma that

$$
\left\|f_{m+1}-f_{m}\right\|_{1} \leq \frac{K}{2^{m} \cdot\left(1-\lambda_{m+1}\right)} \cdot\left\|f_{m}\right\|_{1} .
$$

We now choose a sequence $\lambda_{m}$ which goes fast enough to 1 to get a wandering domain but not too fast to keep control of the $C^{1}$ norm: for instance $\lambda_{m}=$ $1-1 /(m+1)^{2}$.

We get

from $(* *)$; thus,

$$
\left\|f_{m+1}-f_{m}\right\|_{1} \leq K \cdot \frac{(m+2)^{2}}{2^{m}} \cdot\left\|f_{m}\right\|_{1}
$$

$$
\left\|f_{m+1}\right\|_{1} \leq \prod_{i=1}^{m}\left(1+\frac{K \cdot(i+2)^{2}}{2^{i}}\right) \cdot\left\|f_{1}\right\|_{1} .
$$

The product $\prod_{i=1}^{m}\left(1+\left(K \cdot(i+1)^{2}\right) / 2^{i}\right)$ converges to a finite limit as $m \rightarrow \infty$, as easily follows from $\prod_{k=0}^{\infty}\left(1+x^{2^{k}}\right)=1 /(1-x)$ for $|x|<1$. Consequently $\left\|f_{m}\right\|_{1}$ is uniformly bounded by a constant $C>0$. Using $(* *)$ again, we have

$$
\left\|f_{m+1}-f_{m}\right\|_{1} \leq C \cdot K \cdot(m+2)^{2} / 2^{m} \text {. }
$$

It follows that $\left\{f_{n}\right\}_{n}$ is a Cauchy sequence in the $C^{1}$ topology and, hence, converges to a $C^{1}$ limit map. Throughout the construction, one can control that the inverse maps $f_{n}^{-1}$ converge as well in the $C^{1}$ topology so that $f$ is a $C^{1}$ diffeomorphism of $\mathbb{D}^{2}$. 
Let

$$
K=\bigcap_{m \geq 0} \bigcup_{i=0}^{2^{m}-1} f^{i}\left(D_{m}\right)
$$

Then $f$ is a $C^{1}$ infinitely renormalizable diffeomorphism and $(K, f)$ is of type $\left\{a_{n}\right\}_{n}$ with $a_{n} \equiv 2$. Since $\bigcap_{m \geq 0} D_{m}$ is a disk with radius $\prod_{m=1}^{\infty} \lambda_{m}=$ $\prod_{k=2}^{\infty}\left(1-1 / k^{2}\right)=\frac{1}{2}>0, f$ has a wandering domain. Finally, since in this construction, we have

$$
\forall m \geq 1, \quad \delta_{m+1,1}<\delta_{m, 1} ;
$$

is is obvious that $(K, f)$ has a 1-bounded geometry.

\section{ACKNOWLEDGMENT}

We thank Alec Norton for pointing out a mistake in the first version of this text, and the referee for assistance in clarifying the exposition.

\section{REFERENCES}

[B] P. Bohl, Uber die hinsichtlich der unabhängigen variabeln periodische Differentialgleichung erster Ordnung, Acta Math. 40 (1916), 321-336.

[BF] R. Bowen and J. Franks, The periodic points of maps of the disk and the interval, Topology 15 (1976), 337-342.

[D] A. Denjoy, Sur les courbes définies par les équations différentielles à la surface du tore, J. Math. Pures Appl. (9) 11 (1932), 333-375.

[GST] J. M. Gambaudo, S. van Strien, and C. Tresser, The periodic orbit structure of orientation preserving diffeomorphisms on $\mathbb{D}^{2}$ with topological entropy zero, Ann. Inst. H. Poincare Phys. Théor. 49 (1989), 335-356.

[S] D. Sullivan, Bounds, quadratic differentials, and renormalization conjectures, Mathematics into the Twenty-first Century, Vol. II, Amer. Math. Soc. Centennial Publication, Amer. Math. Soc., Providence, RI, 1992, pp. 417-466.

(C. Bonatti and J. M. Lion) Laboratoire De Topologie, B.P. no. 138, 21004 Dijon CedeX, FRANCE

(J. M. Gambaudo) Institut Non-linéaire de Nice, Faculté des Sciences, 06018 Nice CedeX 2, FrANCE

(C. Tresser) I.B.M., PO Box 218, Yorktown Heights, New York 10598 